Check for updates

Cite this: J. Mater. Chem. A, 2017, 5, 24686

Received 7th April 2017

Accepted 6th November 2017

DOI: $10.1039 / c 7 t a 03027 d$

rsc.li/materials-a

\section{Graphitic nanostructures in a porous carbon framework significantly enhance electrocatalytic oxygen evolution $\uparrow$}

\author{
Srinivas Gadipelli, (D) *a Zhuangnan Li, ${ }^{\text {a }}$ Tingting Zhao, ${ }^{a}$ Yuchen Yang, ${ }^{a}$ Taner Yildirim ${ }^{b}$ \\ and Zhengxiao Guo (iD) ${ }^{a}$
}

A hybrid structure, a graphitic nanostructuresaporous carbon framework, is developed by utilizing the bimetallic zeolitic imidazolate framework-8 (ZIF-8) as a solid precursor, simultaneously templating porous carbon and growing graphitic nanocarbon in a simplified chemical vapor deposition (CVD) fashion. A ligand, 2-methylimidazolate (2MIM), in the ZIF-8 decomposes above $600{ }^{\circ} \mathrm{C}$ to yield active carbon/hydrocarbon radicals/vapour. With the idea of using the high catalytic activity of nickel to grow graphitic nanostructures in a CVD process from gaseous carbon feedstocks, a precursor, bimetallic ZIF8 , is synthesized by partial substitution of zinc metal centres by nickel. Such nickel centres thus act as nanocatalysts to grow graphitic nanostructures from the carbon radicals arising from the partly decomposed ligand of the framework during the carbonization step. These hybrid structures show a highly enhanced electrocatalytic activity for the water splitting oxygen evolution reaction (OER). Furthermore the catalytic activity for the oxygen reduction and hydrogen evolution reactions (ORR and $\mathrm{HER}$ ), and gas uptake capacities for $\mathrm{H}_{2}$ and $\mathrm{CO}_{2}$ are enhanced with respect to the increased porosity and nitrogen doping in the samples. We also show that not all the MOF-based precursors with nickel metal centres are suitable for producing nanographitic structures. Our further investigation suggests that the graphitization in the samples plays a critical role in enhancing the catalytic activities.
The design and development of multifunctional porous carbon nanostructures as electrode materials are highly desirable for many electrochemical energy conversion and storage devices, such as fuel-cells, metal-air batteries and supercapacitors..$^{\mathbf{1 - 5}}$ Such multi-functionalities, for example, for guest storage/ accessibility, selective binding, catalysis and charge-transfer, are often associated with local crystallinity and chemistry at the atomic and nano-scales. There have been many attempts to synthesize such structures from a wide variety of carbon precursors with top-down and bottom-up molecular building blocks. ${ }^{2-12}$ Specifically, molecular building blocks as self- and/or sacrificial templates are highly attractive, as those architectures can be made with the choice of a range of multiple heteroatoms and functional groups as well as metal centres. The best examples are biomass, ionic liquids, polymers, solvents and coordinated organic ligands. Often, the precursors containing those heteroatoms (mostly oxygen, nitrogen, sulphur, phosphorus and alkali/alkaline earth metals) can yield highly porous

${ }^{a}$ Department of Chemistry, University College London, 20 Gordon Street, London, WC1H 0AJ, UK. E-mail: gsrinivasphys@gmail.com

${ }^{b}$ NIST Center for Neutron Research, National Institute of Standards and Technology, Gaithersburg, Maryland, 20899, USA

$\dagger$ Electronic supplementary information (ESI) available. See DOI: $10.1039 / \mathrm{c} 7 \mathrm{ta} 03027 \mathrm{~d}$ carbons with dopants. ${ }^{2-12}$ However, the majority of the structures are amorphous in nature, even carbonized at high temperature. This impedes their ability for fast electron/charge transfer in electrochemical devices. ${ }^{13}$ Whereas the highly graphitized structures developed in traditional catalytic CVD (chemical vapour deposition) using gaseous and liquid precursors suffer from cost and/or scaling-up issues due to extensive chemical manipulations. ${ }^{14-16}$ Such CVD carbons also exhibit very poor porosity with fewer active/defective/binding sites, which are essential to enhance their energy conversion and storage capabilities.

On the other hand, predesigned carbon precursors based on metal-organic frameworks (MOFs), a type of coordinated polymer with metal centres, with their highly ordered porous structure are gaining tremendous attention as multifunctional materials for many green energy applications, including gas storage and $\mathrm{CO}_{2}$ capture. ${ }^{5,17-26}$ For instance, depending on the porosity, framework structure, ligand type and metal-centre, MOFs can generate ultrahigh-porosity carbons for highcapacity guest accessibility or gas storage. Indeed, the MOF structures can be synthesized readily at a large scale and are commercially available. ${ }^{27-29}$ In view of electrochemical energy conversion and storage, those MOF precursors can yield suitable supports to bridge and boost the activities of nanoscale 
catalysts and/or active porous carbon hybrid structures, from the self-decomposition and rearrangement of ligand carbons with embedded transition metal-complexes during carbonization. ${ }^{5}$ Furthermore, such hybrid porous carbons, consisting of graphitic nanostructures and with a fine dispersion of foreign atoms to induce surface charge heterogeneity, synergistically enhance their performance in energy storage and conversion. ${ }^{2-30}$ Though there are several available literature reports on MOF-derived carbon nanostructures, there is still a lack of information on how the structure transformations occur under carbonization conditions and also with respect to the particular metal or ligand linker in the framework.

Herein, we designed a simple synthesis technique for the development of porous carbon hybrids (ZNDCs) consisting of catalytic nickel-induced graphitic nanostructures from a bimetallic ZIF-8 (Fig. 1). The main idea behind utilizing ZIF-8 is that it can (1) act as sacrificial template to produce porous carbon by framework decomposition and rearrangement, and vaporization of zinc metal centres, (2) produce nitrogen-doped carbon from its intrinsic framework ligand (2-methylimidazolate, 2MIM) nitrogen, and (3) be a solid precursor to generate carbon radicals from the decomposed ligand to grow highly conducting graphitic nanostructures with its in situ

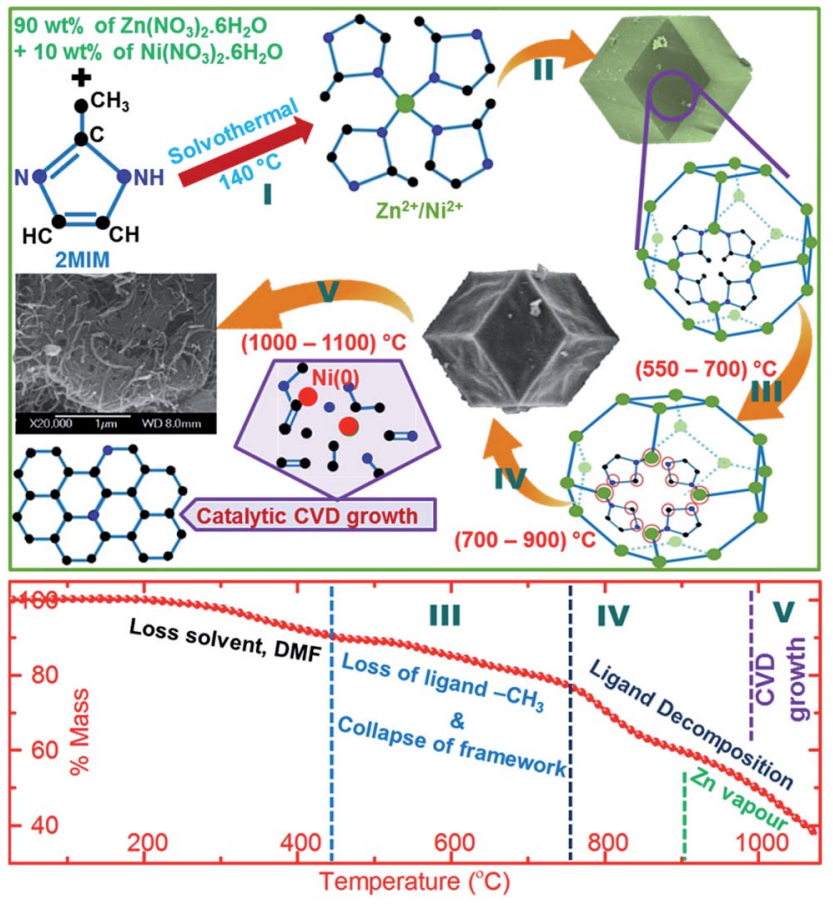

Fig. 1 Schematic representative synthesis of graphitic porous carbon nanostructures: top - from left and in the clockwise direction shows the synthesis of bimetallic ZIF-8 followed by carbonization. The graphitic nanostructure growth can be understood by reduced $\mathrm{Ni}^{2+}$ centres as nanocatalysts for the decomposed-ligand nitrogen and carbon radicals as in a traditional catalytic CVD growth of graphitized structures from the gas-phase carbon precursors. Bottom - TG plot of ZIF- 8 replicates the above mechanism with the representative mass losses due to the decomposition of the framework, and conversion of the ligand into hydrogen, carbon and nitrogen radicals, evaporation of zinc metal centres and CVD growth of nanographites. catalytic transition metal centres in a CVD fashion., $5,15,16,24,31$ Thus, with these points in mind, we have prepared a precursor, nickel-substituted ZIF-8 by a solvothermal method from a mixture of metal salts consisting of $\mathrm{Zn}\left(\mathrm{NO}_{3}\right)_{2} \cdot 4 \mathrm{H}_{2} \mathrm{O}$ and $\mathrm{Ni}\left(\mathrm{NO}_{3}\right)_{2} \cdot 6 \mathrm{H}_{2} \mathrm{O}$ in a $90: 10$ mass ratio (Fig. 1, steps 1-2, Fig. S1 $\dagger$ ). Later, the ZNDCs are obtained by direct pyrolysis of bimetallic ZIF-8 at elevated temperatures without the addition of extra heteroatoms or metal-complexes (Fig. 1, steps 3-5). In a controlled experiment, carbon derived from ZIF-8 alone is predominantly amorphous in nature. Electrochemical studies, under basic conditions, reveal that the ZNDCs with nanographites exhibit a considerable enhancement in their electrocatalytic activity for the oxygen evolution reaction (OER). Furthermore, we also found direct porosity-enhanced lowpressure hydrogen and carbon dioxide uptake capacities. The results clearly suggest overall improvements in the OER, ORR (oxygen reduction reaction) and HER (hydrogen evolution reaction) catalytic activity of ZNDCs with respect to the increased graphitization. A clear influence of doped nitrogen functionality is seen in the ORR activity, and hydrogen and carbon dioxide uptake. The development of nanographites also enhances the stability of the OER. Finally, we also show that not all the MOF-based precursors with nickel metal centres are suitable for generating nanographitic structures in their selftemplated porous carbon framework.

The as-synthesized precursor samples, ZIF-8 and bimetallic ZIF-8, are isostructural in nature (Fig. 1, S2 and $3 \uparrow$ ). Thermogravimetric (TG) analysis of the samples shows four prominent mass-loss regions with respect to temperature. Initial mass-loss in the region up to $400{ }^{\circ} \mathrm{C}$ is due to the evaporation of poreoccluded solvent. Subsequent mass-loss regions, (500-700) ${ }^{\circ} \mathrm{C}$, (700-900) ${ }^{\circ} \mathrm{C}$ and $>900{ }^{\circ} \mathrm{C}$ are mainly attributed to the loss of free rotational methyl groups, framework and ligand decomposition, and zinc evaporation, respectively. Framework and ligand decomposition of ZIF-8 has been well analysed and documented with the results of PXRD (powder X-ray diffraction), XPS (X-ray photoemission spectroscopy), FTIR (Fourier transformed infrared spectroscopy), porosity (specific surface area and micropore volume), gas (hydrogen and carbon dioxide) uptake and combined TG-MS (mass spectroscopy) measurements. ${ }^{5,24,31}$ In particular, carbonization at a temperature above $600{ }^{\circ} \mathrm{C}$ results in the evolution of hydrogen, nitrogen and their hydrocarbon mixtures from the recombination of the decomposed-ligand active hydrogen, carbon and nitrogen radicals. Such decomposition is an ideal source of carbon for the in situ growth of graphitized nanostructures on the reduced nickel catalytic nanocentres, very similar to the catalytic CVD process, but without the use of the dangerous/explosive gaseous hydrocarbon feedstocks. Therefore our rationally designed zinc-nickel-based bimetallic ZIF-8 precursor can serve multiple roles, as (1) self-contained catalyst nickel, (2) a solid-state carbon precursor, (3) a self-template, to obtain highly graphitized nanocarbons, in a CVD manner, within the porous carbon framework, reconstructed from its sacrificial framework template, and (4) a well-controlled method, suitable for largescale synthesis and safe handling. 
To validate the above idea, we have carried out the carbonization of ZIF-8 and bimetallic ZIF-8, separately at discrete temperatures between 700 and $1100{ }^{\circ} \mathrm{C}$ in a tube furnace, under only nitrogen atmosphere without a supply of external hydrogen and hydrocarbon feedstocks. The thus-obtained carbons (named ZDCs and ZNDCs, respectively, derived from ZIF-8 and bimetallic ZIF-8) are characterized without further chemical treatment, such as acid washing. SEM and TEM micrographs show the clear growth of a graphitic nanostructures@amorphous type porous carbon framework in the ZNDCs, synthesized at $\geq 1000{ }^{\circ} \mathrm{C}$ (Fig. 2 and S4-S6†). Here, it is worth noticing that such nanographitic growth is not observed in ZDCs derived from ZIF-8 alone under similar synthetic conditions (Fig. S7 $\dagger$ ). Thus, our idea of partial substitution of nickel for zinc in ZIF-8 yielded a hybrid carbon structure consisting of nanographites within the self-templated porous

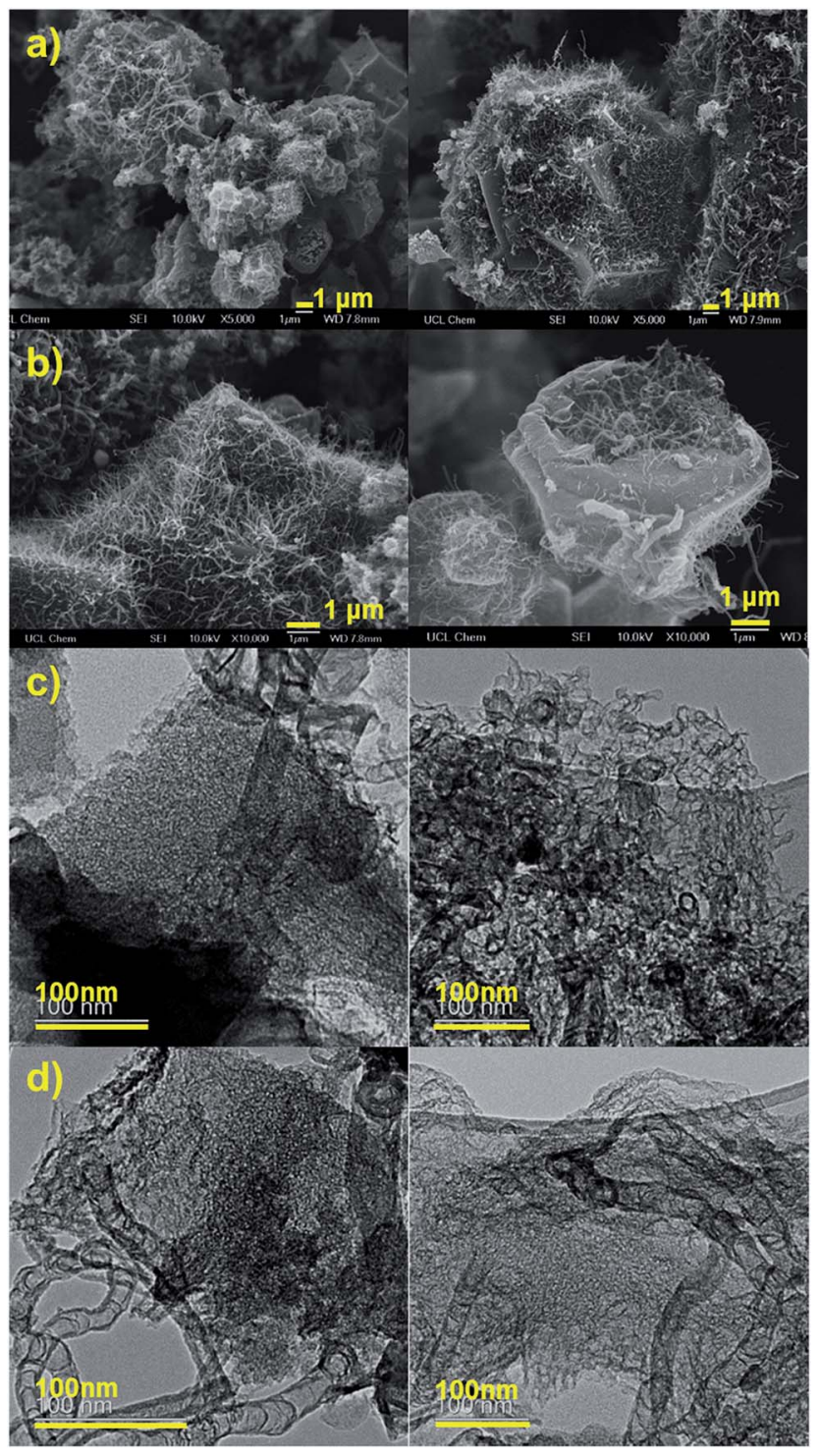

Fig. 2 Surface morphology and growth of graphitic nanostructures in the porous carbon framework of ZNDC samples: SEM $(a, b)$ and TEM (c, d) micrographs of ZNDC1000 (a, c) and ZNDC1100 (b, d). carbon framework. A clear temperature-induced enhancement of nanographite growth is observed. This can be directly attributed to the generation of more active carbon radicals and their solubility in the catalytic nickel centres (an enhanced thermal induced fluidity), similar to the high-temperature catalytic cracking of hydrocarbons or graphenic film growth on foils/wire-meshes. ${ }^{\mathbf{1 4 - 1 6}}$ Indeed, TEM images of the graphitic nanostructures in our samples resemble tubular graphenic structures. Varied dimensions in length and diameter can be directly attributed to the different cluster sizes of the nickel centres.

The framework decomposition followed by graphitization is further well supported by XPS and PXRD (Fig. 3a-c and S8†). It is more striking that $\mathrm{C} 1 \mathrm{~s}$ and $\mathrm{N} 1 \mathrm{~s}$ spectra show a considerable loss of framework nitrogen and enhanced graphitization with an increasing carbonization temperature above $700{ }^{\circ} \mathrm{C}$. Graphitization can be understood by the gradual narrowing and peak shift of $\mathrm{C}$ 1s spectra compared to the pure-phase graphitic structure at $\sim 284.6 \mathrm{eV} .^{5,25}$ For instance, the $2 \mathrm{MIM}$ in the ZIF-8 structure exhibits a C 1s peak, positioned at $\sim 285.3 \mathrm{eV}$. The nitrogen doping into the carbon matrix is seen with substantial changeover in the $\mathrm{N}$ 1s spectra, exhibiting pyridinic $(\sim 398 \mathrm{eV})$ and graphitic $(\sim 401 \mathrm{eV})$ type nitrogen from one type of nitrogen in the starting ZIF-8 sample. Zn 2p spectra further support the

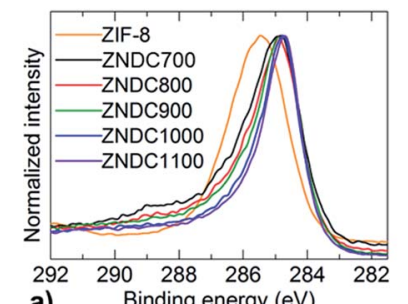

a)
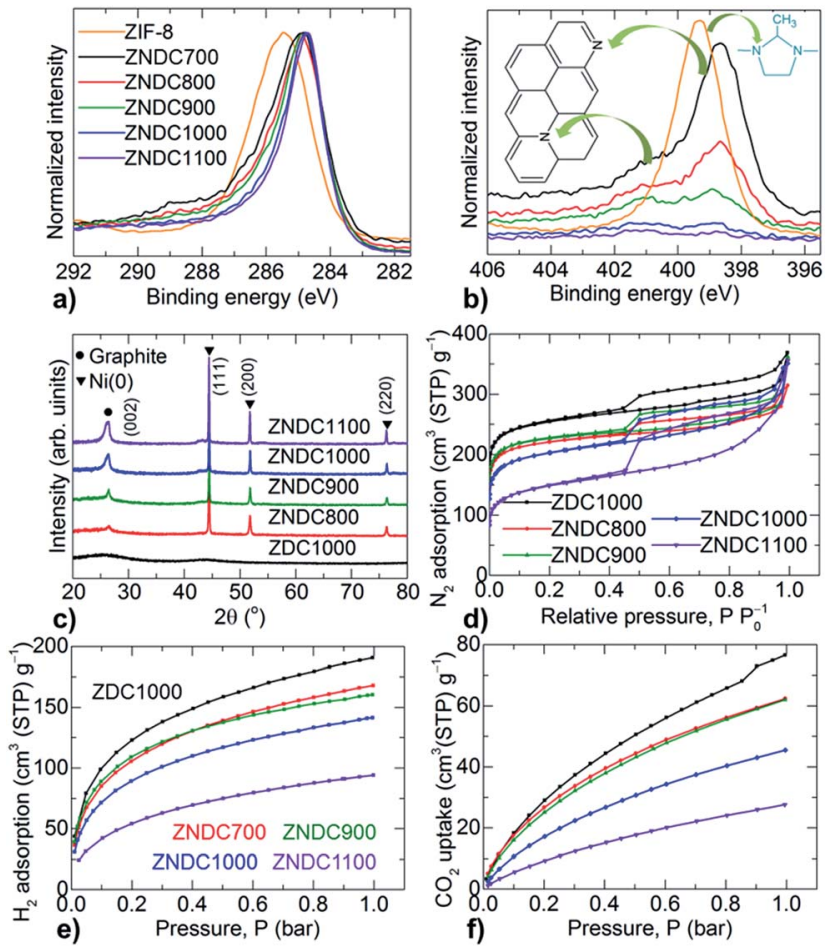

Fig. 3 Structural characteristics of the ZNDC and ZDC samples derived at different temperatures: ( $a, b)$ XPS C 1s (a) and N 1s (b) spectra. Precursor ZIF- 8 is also measured to show ligand decomposition followed by graphitization. (c) PXRD patterns. (d) Porosity estimation $\mathrm{N}_{2}$ adsorption-desorption isotherms, measured at $77 \mathrm{~K} .(\mathrm{e}, \mathrm{f})$ Structure and porosity induced gas uptake behaviour of ZNDCs: $\mathrm{H}_{2}$ uptake at $77 \mathrm{~K}(\mathrm{e})$ and $\mathrm{CO}_{2}$ uptake at $298 \mathrm{~K}$ (f) isotherms. The same colour code applies for $(a, b)$ and $(e, f)$. 
complete evaporation of zinc metal at $>900{ }^{\circ} \mathrm{C}$. PXRD patterns reveal only crystalline reduced nickel centres and enhancement in the nickel-induced graphitization at an increased temperature of carbonization. Note that ZDCs derived fom ZIF-8 alone are amorphous in nature. ${ }^{24}$ Furthermore, the nickel-assisted temperature-induced formation of nanographites is also observed by a continuous decrease in the porosity of the ZNDC samples (Fig. 3d and S9†). Otherwise, ZDCs exhibit considerable enhancement in their porosity with an increased carbonization temperature., ${ }^{54}$

Hydrogen and carbon dioxide adsorption isotherms measured at $77 \mathrm{~K}$ and $298 \mathrm{~K}$, respectively, show a clear basicityenhanced uptake in the samples due to the active defects of the decomposed framework and doped nitrogen functionality (Fig. 3e, f and S10†). A high amount of defective sites generated from the broken $\mathrm{Zn}-\mathrm{N}$ links at a low carbonization temperature $\left(700{ }^{\circ} \mathrm{C}\right.$ ), a framework decomposition point (before actual ligand decomposition), leads to a high $\mathrm{CO}_{2}$ uptake capacity at low partial pressures. ${ }^{31} \mathrm{~A}$ two-step mass-loss behaviour in the TG, under an oxidative atmosphere, indicates the hybrid nature of the ZNDC1100 sample, i.e., a graphitic nanostructures@porous carbon framework in an $\sim 1: 1$ mass ratio (Fig. S11†). Where the porous carbon framework in its amorphous state exhibits less thermal stability compared to that of the nickel-induced crystalline graphite. The TG plot also estimates about 11 mass\% of nickel in the sample.

Next, the structure-property, electrochemical energy conversion relationships of these ZNDCs are investigated by studying their characteristic performance in OER, HER and ORR under alkaline electrolyte (Fig. 4 and S12†). A significant enhancement in their OER activity is seen with respect to the increased growth of nanographites in the hybrid samples (Fig. 4a). Note that an amorphous ZDC1000 sample shows comparatively very poor OER activity. The enhanced activity in the graphitized hybrid catalysts can be understood from the high response of current density, at relatively lower applied potentials. For example, to achieve a benchmark OER response current density of $10 \mathrm{~mA} \mathrm{~cm}{ }^{-2}$, our ZNDC1100 sample required $<1.67 \mathrm{~V}$ ( vs. RHE, an overpotential of $<0.44 \mathrm{~V}$ ), which is lower than the reference $\mathrm{IrO}_{2} / \mathrm{C}$ standard. ${ }^{32}$ Continuously improved OER activity of the ZNDC samples is in good agreement with the temperature-induced growth of nanographites, as confirmed from the SEM, TEM, PXRD, XPS and porosity characteristics.

Nanographitic hybrid samples also show a considerable improvement in their HER activity when compared to their less graphitized and amorphous counterparts (Fig. S12†). ORR activity plots reveal interesting results, where the samples carbonized at lower temperatures (800-1000) ${ }^{\circ} \mathrm{C}$ with more nitrogen doping content and/or porosity exhibit a favourable onset reduction potential and high current density response (Fig. S12†).,5,19,20,33-36 Whereas, the increased growth of nanographites leads to steep oxygen reduction slopes attributed to fast electron transfer and reduction kinetics. Furthermore, the hybrids, ZNDC1100 and ZNDC1000 samples, show a highly enhanced bifunctional OER and ORR activity (a potential difference to achieve the corresponding benchmark current densities of $-3 \mathrm{~mA} \mathrm{~cm}^{-2}$ and $10 \mathrm{~mA} \mathrm{~cm}^{-2}$, respectively for ORR
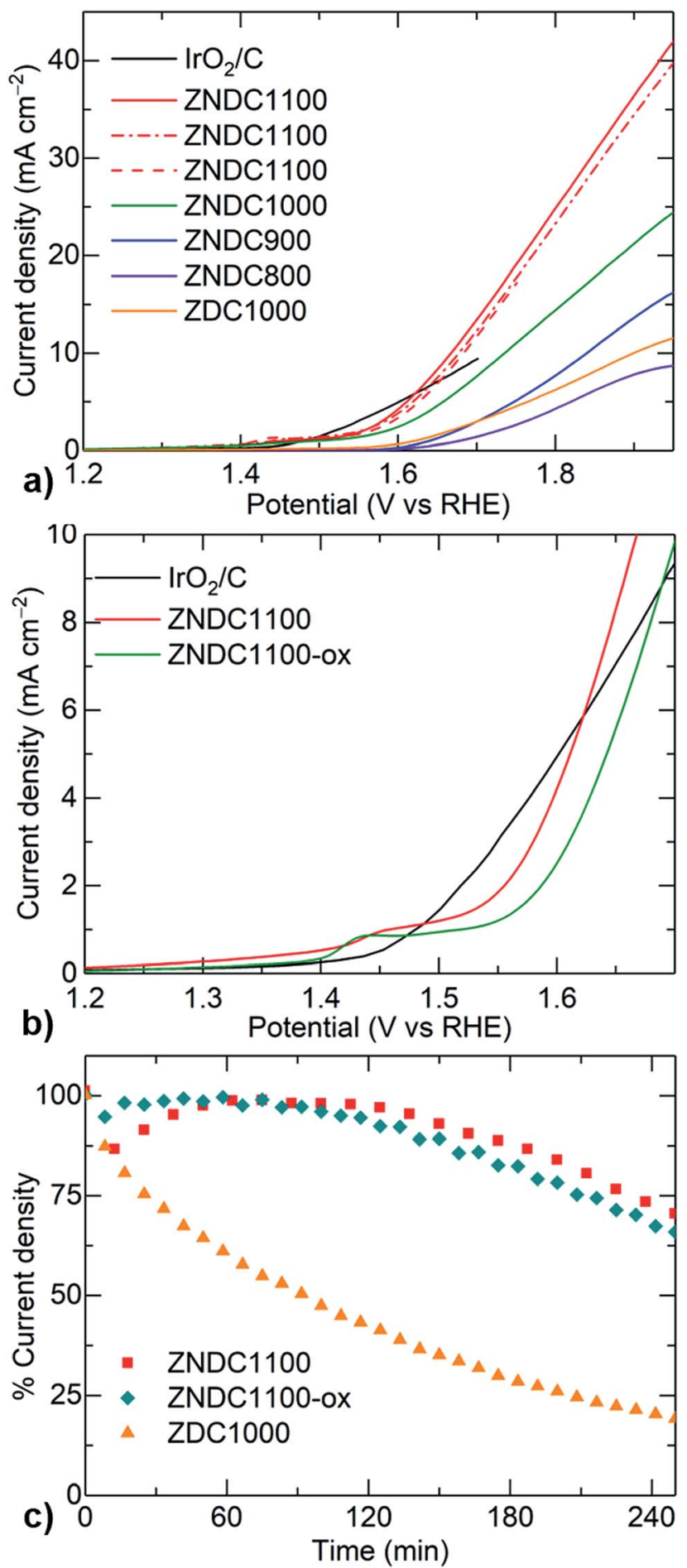

Fig. 4 Electrocatalytic activity of the drop-cast ZNDC and ZDC samples onto a glassy carbon electrode (GCE) disk, measured in a three-electrode cell under $0.1 \mathrm{M} \mathrm{KOH}$ electrolyte: (a) OER linear sweep voltammogram (LSV) curves at $1600 \mathrm{rpm}$. OER performance data on the ZNDC1100 sample is reported from three freshly prepared sample electrodes. (b) Comparative OER LSV curves of ZNDC1100 and ZNDC1100-ox. (c) CA stability data for OER at $1.65 \mathrm{~V}$ (vs. RHE), recorded after stabilizing for $5 \mathrm{~min}$. Reference sample, $\mid \mathrm{rO}_{2} / \mathrm{C}$ in $(\mathrm{a}, \mathrm{b})$ is reproduced from ref. 32 . All the LSV curves are recorded at a scan rate of $10 \mathrm{mV} \mathrm{s}^{-1}$. 
and OER), compared to their amorphous counterpart, the ZDC1000 sample (see Table in Fig. S12 $\dagger$ ). For example, the ZNDC1000 sample exhibits a considerably smaller potential difference of $\sim 1.10 \mathrm{~V}$ compared to the ZDC1000 sample with $\sim 1.43 \mathrm{~V}$.

We have also investigated the catalytic activity of ZNDC1100 after oxidation in a controlled atmosphere to yield NiO@Ni in the hybrid (ZNDC1100-ox). PXRD, XPS and porosity measurements reveal a partial surface oxidation of the nickel, i.e., $\mathrm{NiO} @ \mathrm{Ni}$ and reduction in the porosity due to partial burning of the amorphous carbon (Fig. S13†). Comparative OER and ORR data shows reduced activity in the oxidized sample, ZNDC1100ox, with respect to the ZNDC1100 (Fig. 4b and S14†).37,38 Furthermore, the TG of the ZNDC1100-ox sample under an oxidative atmosphere estimates the nickel concentration to be $\sim 35$ mass\%, much higher than the $\sim 11$ mass\% in the ZNDC1100 sample (Fig. S15†). Also, ZNDC1100-ox exhibits predominantly graphitized carbon. Our earlier studies show that a high concentration of metal content in the graphitized carbon has a detrimental effect on their OER and ORR catalytic activity. ${ }^{5}$ This is mainly attributed to the poor dispersion of the highly agglomerated metal centres and intense graphitization, leading to a considerably smeared-out active surface area for catalysis. In addition, the chronoamperometry (CA) response (current density $v s$. time plots at a constant applied potential) reveals a substantially improved OER stability in the highly graphitized samples (ZNDC1100 and ZNDC1100-ox) compared to the amorphous counterpart, ZDC1000 (Fig. 4c).

Finally, we note that the framework structure and ligand decomposition in the precursor MOF structures is the key to the successful growth of graphitic nanostructures in a CVD fashion. To validate this, we have synthesized a precursor Ni-MOF-74, which has been developed from the carboxylate-based linker, 2,5-dihydroxyterephthalalic acid, to exhibit one-dimensional pore channels (Fig. 5 and S16†)..$^{22,25}$ Surprisingly, the carbonized sample (MDC1000) does not reveal any sign of the development of graphitic nanostructures as observed in nickeldoped ZIF-8. This could be directly attributed to the lower tendency of decomposition of the benzene ring compared to the imidazole ring with volatile nitrogen, as observed from the high temperature TG under inert atmosphere (Fig. 5a). The structure-bridging oxygen groups between the linker and the metal centres result in the formation of metal-oxide clusters and gasification of ligand carboxylates in the form of $\mathrm{CO}_{2} .^{22,24,25,39,40}$ With increasing temperature, the crystalline metal-oxide in the carbon matrix gradually reduces to the metal, $2 \mathrm{NiO}+\mathrm{C} \rightarrow 2 \mathrm{Ni}+$ $\mathrm{CO}_{2}$ (gas). ${ }^{25}$ Thus, this process could be the inhibiting factor for the actual catalytic activity of nickel to yield the graphitic nanostructures (Fig. 5 and S17 $\dagger$ ). ${ }^{22,41}$ Also a large clustering of nickel can be seen in the SEM micrographs. After acid washing to remove the nickel (MDC1000-aw), the sample exhibits a mesoporous nature with a large pore type, similar to the other salt-templated carbons (Fig. 5b-f, S16 and S17†). Such large clusters of nickel are another reason nanographites are not produced; instead they yield graphitic shells. ${ }^{14,15,22,42}$ On the other hand, during the carbonization of ZIF-8, which is an oxygen-free structure, the metal centres are stabilized by the
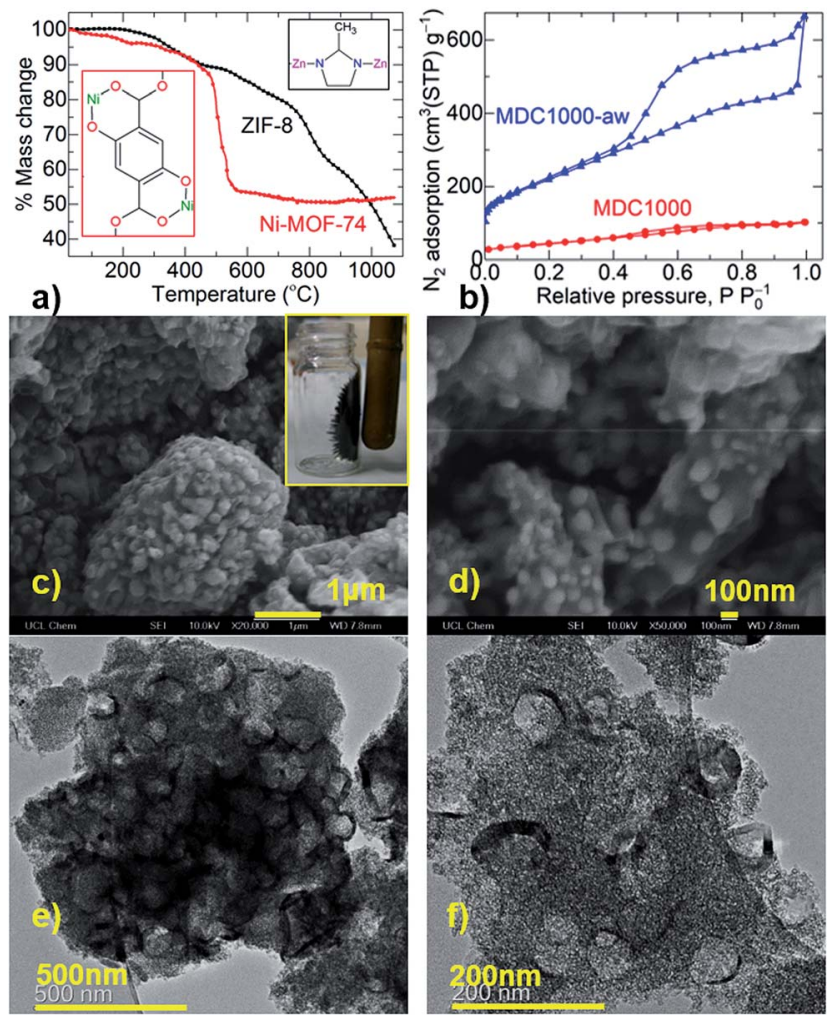

Fig. 5 Synthesis and characteristics of Ni-MOF-74 derived carbons: (a) TG curves of Ni-MOF-74 and ZIF-8 samples with inset framework linkers. (b) $\mathrm{N}_{2}$ adsorption-desorption isotherms (at $77 \mathrm{~K}$ ) of MDCs, giving a specific surface area and total pore volume of 150 and $710 \mathrm{~m}^{2} \mathrm{~g}^{-1}$ and 0.16 and $1.03 \mathrm{~cm}^{3} \mathrm{~g}^{-1}$, respectively, for the samples, before and after acid washing. (c, d) SEM micrographs of MDC1000, with inset photograph showing magnetic behaviour of the reduced nickel centres in the carbon shell. (e, f) TEM micrographs of MDC1000-aw, showing a porous carbon nature with graphitic shells formed around nickel clusters (etched by $\mathrm{HCl}$ acid).

nitrogen atoms within the carbon matrix. ${ }^{5}$ Therefore, ZIF-8 acts as an ideal solid state precursor for the transition metal-based catalytic CVD growth of the nanographites. Also, this method is very safe (as no explosive feedstocks or sophisticated/ expensive commercial CVD equipment is required) and the product can be scaled-up by utilizing such kinds of precursors.

Electrochemical studies on the MDC1000-aw sample further support the important role of the nanographites and nitrogen dopants to enhance the OER activity in the carbon-based samples (Fig. S18 $\dagger$ ). For instance, despite having a higher specific surface area $\left(710 \mathrm{~m}^{2} \mathrm{~g}^{-1}\right.$, compared to $550 \mathrm{~m}^{2} \mathrm{~g}^{-1}$ in ZNDC1100), the MDC1000-aw sample shows a relatively low OER activity. Obviously, the MDC1000-aw sample exhibits a low degree of graphitization and contains no nitrogen dopants. It is worth noting that our hybrid sample, ZNDC1100, synthesized in a very simplified manner shows the best OER activity compared to carbons synthesized with extensive chemical manipulation in the literature (Fig. S19†)., ${ }^{2,3,711,12,43}$ For example, a nitrogen, phosphorus, and fluorine tri-doped graphene sample synthesized at $950{ }^{\circ} \mathrm{C}$ still exhibits poor OER performance, and required $1.82 \mathrm{~V}$ ( $v s$. RHE), compared to $1.67 \mathrm{~V}$ ( $v s$. RHE) for our 
ZNDC1100, to achieve a benchmark current density of $10 \mathrm{~mA} \mathrm{~cm}^{-2} \cdot{ }^{12}$ Our ZNDC1100 sample also shows a considerably improved OER activity over dual-doped nitrogen and sulphur and/or nitrogen and metal@doped nanocarbons., ${ }^{2,3,7,43}$

Furthermore, our literature data mining plot suggests that the carbons in their pristine and/or oxidative state (which includes graphene-, carbon nanotube- and ordered mesoporous- or hierarchical porous carbon-based structures) are very poor OER performers (Fig. 6 and S20†). However, their activity has been considerably enhanced with the introduction of metalfree heteroatoms, such as nitrogen, sulphur and phosphorous alone or in combination, dual- or tri-doped state (Fig. 6 and $\mathrm{S} 21 \dagger)$. There is hardly any evidence that dual- or tri-doped carbon structures show superior OER activity compared to singly-doped ones. Overall, it is conceivable that the carbon structures in a combination of nonprecious transition metal (or metal-oxide) centres and heteroatom dopants show greatly enhanced OER activity (Fig. 6 and S20-S22†). Yet, the samples, in each case either in their pristine, oxidative, doped or metal coordinated state, show a considerable discrepancy in their activity response. This could be directly attributed to the inherent structural features of the host carbon itself. For example, graphitization, specific surface area, porosity and pore-size distribution all contribute to the activity in terms of facilitating guest accessibility and transport (ions in the case of OER and HER) or adsorption (in the case of ORR), charge

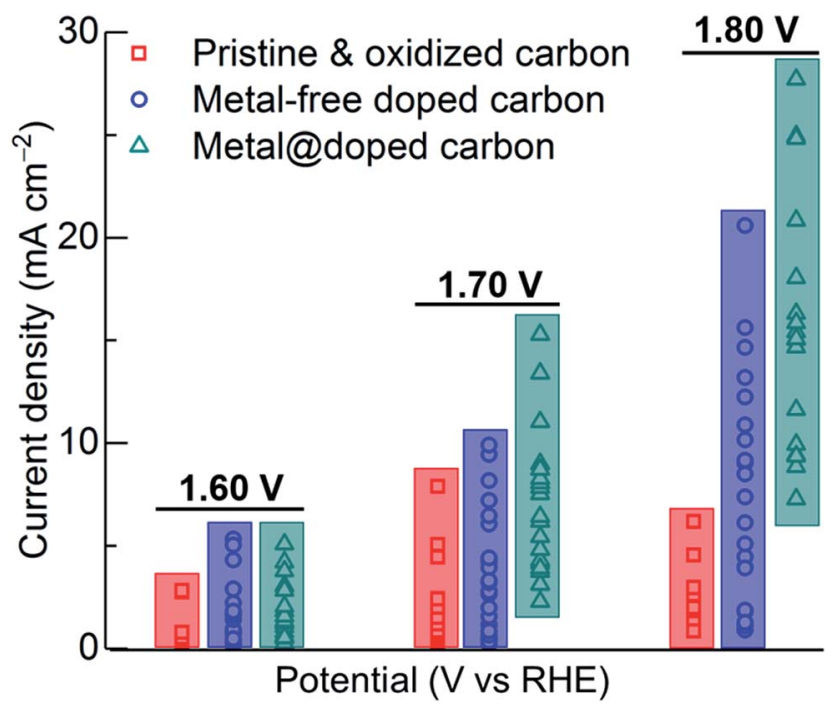

Fig. 6 OER performance data - current density against applied potential (vs. RHE) in a $0.1 \mathrm{M} \mathrm{KOH}$ electrolyte, derived from a variety of carbon-based structures, which include pristine and oxidized carbons, metal-free-heteroatom doped carbons, and metal-embedded doped carbons, reported in the literature (see the additional plots and references in $\mathrm{ESI} \dagger$ ). A wide range of host carbons are used, such as carbonized polymers and MOFs at high temperatures, templated ordered mesoporous carbons, carbon nanotubes and/or combinations of them, thus yielding a large discrepancy in the OER activity response current density for the same given applied voltage. Notably, in the same category (grouped as pristine and/or oxidative, metal-free doped, metal(adoped) one can see a range of responses, from absolutely no activity from one structure to the exceptional activity of another structure. transfer, active surface area and catalytic sites. From the survey it is understood that the samples in a mesoporous state and finely dispersed transition metal centres stabilized with the heteroatom dopants and the metal-induced graphitic, i.e., crystalline carbons in a porous carbon matrix, exhibit outstanding OER activity. Carbon samples in a highly porous state or a graphitized state alone without dopants or metal-/ metal-oxide nanocentres do not yield a high enough catalytic activity response.

\section{Conclusions}

We have shown a new approach to designing a graphitic nanostructures@amorphous porous carbon framework from a single-step, solid-state precursor route, CVD method, in a highly simplified manner by designing a bimetallic (zincnickel) ZIF-8. The oxygen-free ligand, its decomposition into active carbon radicals and highly stabilized nickel nanocentres are the key factors for producing carbon-based hybrid structures. More importantly, our results suggest that the carbon samples, balanced with graphitic nanostructure and porosity, are ideal candidate materials to achieve the best energy conversion and storage abilities. In addition, their overall efficiency for the electrocatalytic activity can be enhanced with dopants, both metallic and nonmetallic implants. Similarly, nitrogen doping and porosity also play a critical role in synergistically enhancing both the catalytic activity and gas uptake at very low-partial pressures.

\section{Experimental}

\section{Synthesis of ZIF-8 and Zn/Ni-ZIF-8}

In a typical synthesis of $\mathrm{Zn}-\mathrm{ZIF}-8,4.666 \mathrm{~g}$ of $\mathrm{Zn}\left(\mathrm{NO}_{3}\right)_{2} \cdot 4 \mathrm{H}_{2} \mathrm{O}$ and $1.333 \mathrm{~g}$ of 2-methyl imidazole were dissolved in $400 \mathrm{ml}$ of $\mathrm{N}, \mathrm{N}$ dimethylformamide (DMF). ${ }^{44}$ The solution was distributed to 5 $\times 100 \mathrm{ml}$ Teflon-lined autoclaves and left in an oven at $140{ }^{\circ} \mathrm{C}$ for $24 \mathrm{~h}$. After cooling down to room temperature, the mother liquor was removed and washed with DMF by centrifugation and dried in an oven at $80{ }^{\circ} \mathrm{C}$. The $\mathrm{Zn} / \mathrm{Ni}-\mathrm{ZIF}-8$ sample was synthesized by a similar method but using a zinc and nickel salt mixture in a $90: 10$ mass ratio: $4.200 \mathrm{~g}$ of $\mathrm{Zn}\left(\mathrm{NO}_{3}\right)_{2} \cdot 4 \mathrm{H}_{2} \mathrm{O}$ and $0.467 \mathrm{~g}$ of $\mathrm{Ni}\left(\mathrm{NO}_{3}\right)_{2} \cdot 6 \mathrm{H}_{2} \mathrm{O}$.

\section{Synthesis of Ni-MOF-74}

$0.478 \mathrm{~g}$ of 2,5-dihydroxyterephthalic acid and $2.378 \mathrm{~g}$ of $\mathrm{Ni}\left(\mathrm{NO}_{3}\right)_{2} \cdot 6 \mathrm{H}_{2} \mathrm{O}$ were dissolved in $200 \mathrm{ml}$ of DMF, ethanol and water in $1: 1: 1(\mathrm{v} / \mathrm{v} / \mathrm{v}) .^{45,46}$ The solution was transferred to a $500 \mathrm{ml}$ wide-mouth screw-cap glass jar and left in an oven at $100{ }^{\circ} \mathrm{C}$ for $24 \mathrm{~h}$. After cooling down to room temperature, the mother liquor was removed and washed with DMF by centrifugation and dried in an oven at $80{ }^{\circ} \mathrm{C}$.

\section{Synthesis of ZDCs, ZNDCs and MDCs}

In a carbonization process, a suitable amount, between 100 and $300 \mathrm{mg}$, of Zn-ZIF-8 or Zn/Ni-ZIF-8 was placed in an alumina boat and then transferred into a horizontal tube furnace. The 
furnace tube was closed with gas feedthrough end seals and the sample area was thoroughly purged with nitrogen. The nitrogen flow was maintained throughout the reaction. The carbonization between 700 and $1100{ }^{\circ} \mathrm{C}$ was carried out for $10 \mathrm{~h}$ at a given temperature after heating at $5{ }^{\circ} \mathrm{C}$ per minute. The carbons obtained are named as ZDCs, ZNDCs, and MDCs, respectively, for ZIF-8, Zn/Ni-ZIF-8, and Ni-MOF-74. Samples synthesized at different temperatures are identified by a suffix number, for example $1000{ }^{\circ} \mathrm{C}$ carbonized samples are named ZDC1000 or ZNDC1000. All the samples for further characterizations were handled in ambient air without further chemical treatment or acid washing unless stated. Nickel etching of the as-synthesized MDC1000 sample was carried out by stirring the sample under concentrated $\mathrm{HCl}$ at room temperature, followed by washing with deionized water and drying at $80{ }^{\circ} \mathrm{C}$. This acid-washed sample is named MDC1000-aw.

\section{Synthesis of ZNDC1100-ox}

The ZNDC1100 sample was oxidized by leaking a small amount of air during the thermal annealing under $\mathrm{N}_{2}$ atmosphere.

\section{Structure \& porosity characterization}

Powder X-ray diffraction (PXRD, on Stoe Stadi-P, Cu-K-alpha) was carried out by filling a $0.5 \mathrm{~mm}$ diameter borosilicate glass capillary with sample under ambient conditions. X-ray photoemission spectroscopy (XPS, on Al-K-alpha, Thermo Scientific) data and scanning electron microscopy (SEM, on JSM6700, Jeol) measurements were carried out on the samples supported on a carbon tape. Transmission electron microscopy (TEM, on Jeol) images were obtained on the samples deposited on a carboncoated copper grid from methanol dispersion. As TEM is not compatible for imaging magnetic samples, the supernatant was pipetted after trapping the magnetic nickel-containing particles in methanol by a strong magnet. The porosity, $\mathrm{N}_{2}$, and $\mathrm{CO}_{2}$ and $\mathrm{H}_{2}$ adsorption-desorption isotherms were measured up to $1 \mathrm{bar}$ at $77 \mathrm{~K}$ and $298 \mathrm{~K}$ using liquid nitrogen and a water bath, respectively, on a Quantachrome Autosorb-iQC. The specific surface area was measured from the $77 \mathrm{~K} \mathrm{~N}_{2}$ isotherm in a relative pressure range between 0.01 and 0.2 , according to the Brunauer-Emmett-Teller (BET) method. The QSDFT (quenched solid density functional theory) method with slit/cylindrical pores was applied to the desorption isotherm $\left(10^{-2}\right.$ to 0.99 , $P P_{\mathrm{o}}{ }^{-1}$ ) to obtain the pore size distribution and cumulative pore volume. All the samples were degassed at $180{ }^{\circ} \mathrm{C}$ overnight under a dynamic vacuum prior to the actual gas adsorption measurements. Combined thermogravimetric (TG, on Setsys from Setaram) analysis and mass spectrometry (MS, on OmniStar from Pfeiffer Vacuum) up to $1080{ }^{\circ} \mathrm{C}$ measurements were carried out under Ar flow with a heating rate of $5{ }^{\circ} \mathrm{C}$ per minute. TG mass-loss was recorded after background correction with an empty alumina crucible.

TG measurements on the ZDC and ZNDC samples were carried out under an oxidative atmosphere, flowing oxygen with a heating rate of $5{ }^{\circ} \mathrm{C}$ per minute up to $800{ }^{\circ} \mathrm{C}$. The nickel mass concentration was estimated from the residual mass after complete burn-off of the carbon.

\section{Electrochemical tests}

All the tests were carried out (on Autolab, Metrohm PGSTAT302N) by a three-electrode method using a glassy carbon rotating disk as the working electrode, and $(1 \times 1) \mathrm{cm}^{2}$ $\mathrm{Pt}$ and $\mathrm{Ag} / \mathrm{AgCl} /$ saturated $\mathrm{KCl}$ as the counter and reference electrodes, respectively. OER and ORR, measurements were carried out in $\mathrm{O}_{2}$-saturated alkaline $(0.1 \mathrm{M} \mathrm{KOH})$ electrolyte at room temperature. HER tests were also measured in $0.1 \mathrm{M} \mathrm{KOH}$. All the measurements were carried out with a fixed catalyst deposition of $\sim 0.28 \mathrm{mg} \mathrm{cm}^{-2}$ on a $3 \mathrm{~mm}$ dia (or area of $0.0707 \mathrm{~cm}^{2}$ ) glassy carbon electrode (GCE). The catalyst was prepared as follows; $2 \mathrm{mg}$ of NPC sample was dispersed in a total $500 \mu \mathrm{l}$ solution consisting $482 \mu \mathrm{l}$ of deionized water plus $18 \mu \mathrm{l}$ of Nafion ( $5 \%$ solution) under sonication. The sonication was carried out for up to an hour to get uniform catalyst dispersion of ink. Of this $5 \mu \mathrm{l}$ was micropipetted and dropped on to a GCE followed by drying at $60{ }^{\circ} \mathrm{C}$ in an oven prior to the electrochemical tests. For ORR, OER and HER, the CV (Cyclic Voltammetry) and LSV (Linear Sweep Voltammetry) curves were recorded with voltage sweeping at $10 \mathrm{mV} \mathrm{s}^{-1}$ in the potential range of $+0.2 \mathrm{~V}$ to $-0.8 \mathrm{~V}$ for ORR, $+0.2 \mathrm{~V}$ to $+1.0 \mathrm{~V}$ for OER, and $-0.8 \mathrm{~V}$ to $-1.8 \mathrm{~V}$ for HER. Before the actual measurements, the catalyst was subjected to a number of CV cycles until a stable CV was obtained. The OER stability $(I v s . t)$ tests were carried out at a fixed potential of $+0.70 \mathrm{~V}$ and the response current recorded against time. The ORR, OER and HER LSV curves were measured at $1600 \mathrm{rpm}$. All the reported current densities were estimated by normalizing the actual current response to the electrode area of GCE. The following relation was used for V ( $v s$. $\mathrm{RHE})=\mathrm{V}(v s . \mathrm{Ag} / \mathrm{AgCl})+0.95 \mathrm{~V}^{37}$ The overpotential was reported according to the relation; $V=V_{\mathrm{RHE}}-1.23 \mathrm{~V}$.

\section{Conflicts of interest}

There are no conflicts to declare.

\section{Acknowledgements}

This work was supported by the EPSRC (Grant No. EP/L018330/1 and EP/K002252/1).

\section{References}

1 V. Dusastre and L. Martiradonna, Materials for sustainable energy, Nat. Mater., 2017, 16, 15.

2 P. Chen, T. Zhou, L. Xing, K. Xu, Y. Tong, H. Xie, L. Zhang, W. Yan, W. Chu, C. Wu and Y. Xie, Atomically dispersed iron-nitrogen species as electrocatalysts for bifunctional oxygen evolution and reduction reactions, Angew. Chem., Int. Ed., 2017, 56, 610.

3 G. Fu, Y. Chen, Z. Cui, Y. Li, W. Zhou, S. Xin, Y. Tang and J. B. Goodenough, Novel hydrogel-derived bifunctional oxygen electrocatalyst for rechargeable air cathodes, Nano Lett., 2016, 16, 6516. 
4 R. C. Raj, A. Samanta, S. H. Noh, S. Mondal, T. Okajimab and T. Ohsaka, Emerging new generation electrocatalysts for the oxygen reduction reaction, J. Mater. Chem. A, 2016, 4, 11156.

5 S. Gadipelli, T. Zhao, S. A. Shevlin and Z. X. Guo, Switching effective oxygen reduction and evolution performance by controlled graphitization of a cobalt-nitrogen-carbon framework system, Energy Environ. Sci., 2016, 9, 1661.

6 S. Gadipelli and Z. X. Guo, Graphene-based materials: synthesis and gas sorption, storage and separation, Prog. Mater. Sci., 2015, 69, 1.

7 J. Wei, Y. Liang, Y. Hu, B. Kong, J. Zhang, Q. Gu, Y. Tong, X. Wang, S. P. Jiang and H. Wang, Hydrothermal synthesis of metal-polyphenol coordination crystals and their derived metal/N-doped carbon composites for oxygen electrocatalysis, Angew. Chem., Int. Ed., 2016, 55, 12470.

8 J. M. Lee, T. Wu, B. M. Alston, M. E. Briggs, T. Hasell, C.-C. Hub and A. I. Cooper, Porosity-engineered carbons for supercapacitive energy storage using conjugated microporous polymer precursors, J. Mater. Chem. A, 2016, 4, 7665.

9 Z. Xu, X. Zhuang, C. Yang, J. Cao, Z. Yao, Y. Tang, J. Jiang, D. $\mathrm{Wu}$ and $\mathrm{X}$. Feng, Nitrogen-doped porous carbon superstructures derived from hierarchical assembly of polyimide nanosheets, Adv. Mater., 2016, 28, 1981.

10 M. Graglia, J. Pampel, T. Hantke, T. P. Fellinger and D. Esposito, Nitro lignin-derived nitrogen-doped carbon as an efficient and sustainable electrocatalyst for oxygen reduction, ACS Nano, 2016, 10, 4364.

11 R. Zhang, C. Zhang and W. Chen, FeP embedded in N, P dual-doped porous carbon nanosheets: an efficient and durable bifunctional catalyst for oxygen reduction and evolution reactions, J. Mater. Chem. A, 2016, 4, 18723.

12 J. Zhang and L. Dai, Nitrogen, phosphorus, and fluorine tridoped graphene as a multifunctional catalyst for selfpowered electrochemical water splitting, Angew. Chem., Int. Ed., 2016, 55, 13296.

13 H. Wang, S. Min, C. Ma, Z. Liu, W. Zhang, Q. Wang, D. Li, Y. Li, S. Turner, Y. Han, H. Zhu, E. Abou-hamad, M. N. Hedhili, J. Pan, W. Yu, K.-W. Huang, L.-J. Li, J. Yuan, M. Antonietti and T. Wu, Synthesis of single-crystal-like nanoporous carbon membranes and their application in overall water splitting, Nat. Commun., 2016, 8, 13592.

14 J. Zhao, H. Lai, Z. Lyu, Y. Jiang, K. Xie, X. Wang, Q. Wu, L. Yang, Z. Jin, Y. Ma, J. Liu and Z. Hu, Hydrophilic hierarchical nitrogen-doped carbon nanocages for ultrahigh supercapacitive performance, Adv. Mater., 2015, 27, 3541.

15 Z. Chen, W. Ren, L. Gao, B. Liu, S. Pei and H.-M. Cheng, Three-dimensional flexible and conductive interconnected graphene networks grown by chemical vapour deposition, Nat. Mater., 2011, 10, 424.

16 S. Gadipelli, I. Calizo, J. Ford, G. Cheng, A. R. H. Walkerb and T. Yildirim, A highly practical route for large-area, single layer graphene from liquid carbon sources such as benzene and methanol, J. Mater. Chem., 2011, 21, 16057.

17 K. Shen, X. Chen, J. Chen and Y. Li, Development of MOFderived carbon-based nanomaterials for efficient catalysis, ACS Catal., 2016, 6, 5887.
18 C. Zhang, Y.-C. Wang, B. An, R. Huang, C. Wang, Z. Zhou and W. Lin, Networking pyrolyzed zeolitic imidazolate frameworks by carbon nanotubes improves conductivity and enhances oxygen-reduction performance in polymer-electrolytemembrane fuel cells, Adv. Mater., 2017, 29, 1604556.

19 C. Liu, J. Wang, J. Li, J. Liu, C. Wang, X. Sun, J. Shen, W. Hana and L. Wang, Electrospun ZIF-based hierarchical carbon fiber as an efficient electrocatalyst for the oxygen reduction reaction, J. Mater. Chem. A, 2017, 5, 1211.

20 L. Li, P. Dai, X. Gu, Y. Wang, L. Yan and X. Zhao, High oxygen reduction activity on a metal-organic framework derived carbon combined with high degree of graphitization and pyridinic-N dopants, J. Mater. Chem. A, 2017, 5, 789.

21 C. Lu, D. Wang, J. Zhao, S. Han and W. Chen, A continuous carbon nitride polyhedron assembly for high-performance flexible supercapacitors, Adv. Funct. Mater., 2017, 27, 1606219.

22 J. A. Carrasco, J. Romero, G. Abellán, J. Hernandez-Saz, S. I. Molina, C. Marti-Gastaldo and E. Coronado, Smallpore driven high capacitance in a hierarchical carbon via carbonization of Ni-MOF-74 at low temperatures, Chem. Commun., 2016, 52, 9141.

23 T. Liu, P. Zhao, X. Hua, W. Luo, S. Chen and G. Cheng, An Fe-N-C hybrid electrocatalyst derived from a bimetalorganic framework for efficient oxygen reduction, J. Mater. Chem. A, 2016, 4, 11357.

24 S. Gadipelli and Z. X. Guo, Tuning of ZIF-derived carbon with high activity, Nitrogen functionality, and yield - a case for superior $\mathrm{CO}_{2}$ capture, ChemSusChem, 2015, 8, 2123.

25 G. Srinivas, V. Krungleviciute, Z. X. Guo and T. Yildirim, Exceptional $\mathrm{CO}_{2}$ capture in a hierarchically porous carbon with simultaneous high surface area and pore volume, Energy Environ. Sci., 2014, 7, 335.

26 S. Gadipelli, H. A. Patel and Z. X. Guo, An ultrahigh pore volume drives up the amine stability and cyclic $\mathrm{CO}_{2}$ capacity of a solid-amine@carbon sorbent, Adv. Mater., 2015, 27, 4903.

27 Y. Pan, H. Li, X.-X. Zhang, Z. Zhang, X.-S. Tong, C.-Z. Jia, B. Liu, C.-Y. Sun, L.-Y. Yang and G.-J. Chen, Large-scale synthesis of ZIF-67 and highly efficient carbon capture using a ZIF-67/glycol-2-methylimidazole slurry, Chem. Eng. Sci., 2015, 137, 504.

28 M. Taddei, D. A. Steitz, J. A. van Bokhoven and M. Ranocchiari, Continuous-flow microwave synthesis of metal-organic frameworks: a highly efficient method for large-scale production, Chem.-Eur. J., 2016, 22, 3245.

29 Y. Chen, S. Li, X. Pei, J. Zhou, X. Feng, S. Zhang, Y. Cheng, H. Li, R. Han and B. Wang, A solvent-free hot-pressing method for preparing metal-organic-framework coatings, Angew. Chem., Int. Ed., 2016, 55, 3419.

30 X. Li, Y. Fang, S. Zhao, J. Wu, F. Li, M. Tian, X. Long, J. Jin and J. Ma, Nitrogen-doped mesoporous carbon nanosheet/ carbon nanotube hybrids as metal-free bifunctional electrocatalysts for water oxidation and oxygen reduction, J. Mater. Chem. A, 2016, 4, 13133.

31 S. Gadipelli, W. Travis, W. Zhou and Z. X. Guo, A thermally derived and optimized structure from ZIF-8 with giant 
enhancement in $\mathrm{CO}_{2}$ uptake, Energy Environ. Sci., 2014, 7, 2232.

32 T. Y. Ma, J. L. Cao, M. Jaroniec and S. Z. Qiao, Interacting carbon nitride and titanium carbide nanosheets for highperformance oxygen evolution, Angew. Chem., Int. Ed., 2015, 55, 1138.

33 S. Lee, D.-H. Kwak, S.-B. Han, Y.-W. Lee, J.-Y. Lee, I.-A. Choi, H.-S. Park, J.-Y. Park and K.-W. Park, Bimodal porous iron/ nitrogen-doped highly crystalline carbon nanostructure as a cathode catalyst for the oxygen reduction reaction in an acid medium, ACS Catal., 2016, 6, 5095.

34 J. Sun, L. Wang, R. Song and S. Yanga, Enhancing pyridinic nitrogen level in graphene to promote electrocatalytic activity for oxygen reduction reaction, Nanotechnology, 2016, 27, 055404.

35 X. Liu, S. Zou and S. Chen, Ordered mesoporous carbons codoped with nitrogen and iron as effective catalysts for oxygen reduction reaction, Nanoscale, 2016, 8, 19249.

36 H.-W. Liang, W. Wei, Z.-S. Wu, X. Feng and K. Müllen, Mesoporous metal-nitrogen-doped carbon electrocatalysts for highly efficient oxygen reduction reaction, J. Am. Chem. Soc., 2013, 135, 16002.

37 J. Wang, K. Li, H. Zhong, D. Xu, Z. Wang, Z. Jiang, Z. Wu and $\mathrm{X}$. Zhang, Synergistic effect between metal-nitrogen-carbon sheets and NiO nanoparticles for enhanced electrochemical water-oxidation performance, Angew. Chem., Int. Ed., 2015, 54, 10530.

38 S. Guo, S. Zhang, L. Wu and S. Sun, Co/CoO nanoparticles assembled on graphene for electrochemical reduction of oxygen, Angew. Chem., 2012, 124, 11940.
39 Q. Zuo, P. Zhao, W. Luo and G. Cheng, Hierarchically porous Fe-N-C derived from covalent-organic materials as a highly efficient electrocatalyst for oxygen reduction, Nanoscale, 2016, 8, 14271.

40 S. Gadipelli and Z. X. Guo, Postsynthesis annealing of MOF-5 remarkably enhances the framework structural stability and $\mathrm{CO}_{2}$ uptake, Chem. Mater., 2014, 26, 6333.

41 J. Kong, A. M. Cassell and H. Dai, Chemical vapor deposition of methane for single-walled carbon Nanotubes, Chem. Phys. Lett., 1998, 292, 567.

42 J. Sha, C. Gao, S.-K. Lee, Y. Li, N. Zhao and J. M. Tour, Preparation of three-dimensional graphene foams using powder metallurgy templates, ACS Nano, 2016, 10, 1411.

43 S. S. Shinde, C.-H. Lee, A. Sami, D.-H. Kim, S.-U. Lee and J.-H. Lee, Scalable 3-D carbon nitride sponge as an efficient metal-free bifunctional oxygen electrocatalyst for rechargeable Zn-air batteries, ACS Nano, 2017, 11, 347.

44 K. S. Park, Z. Ni, A. P. Cote, J. Y. Choi, R. Huang, F. J. UribeRomo, H. K. Chae, M. O'Keeffe and O. M. Yaghi, Exceptional chemical and thermal stability of zeolitic imidazolate frameworks, Proc. Natl. Acad. Sci. U. S. A., 2006, 103, 10186.

45 S. Gadipelli, J. Ford, W. Zhou, H. Wu, T. J. Udovic and T. Yildirim, Nanoconfinement and catalytic dehydrogenation of ammonia borane by magnesiummetal-organic framework-74, Chem.-Eur. J., 2011, 17, 6043.

46 S. R. Caskey, A. G. Wong-Foy and A. J. Matzger, Dramatic tuning of carbon dioxide uptake via metal substitution in a coordination polymer with cylindrical pores, J. Am. Chem. Soc., 2008, 130, 10870. 